\title{
Mapeamento da literatura sobre a relação entre religiosidade, apoio social e atividade física
}

\section{Mapping the literature about the relationship of religiosity, social support and physical activity}

\author{
Mariana Silva dos Reis ${ }^{1,2}$, Maíra Parra ${ }^{3,5}$, Rodrigo Siqueira Reis $1,2,4$
}

\section{Resumo}

Objetivou-se realizar um mapeamento da literatura sobre a relação do apoio social advindo de instituições religiosas com a atividade física. A busca foi realizada em Agosto de 2014, nas bases de dados eletrônicas Medline via Pubmed, EMBASE, CENTRAL (Cochrane Central Register of Controlled Trials), LILACS e PsychINFO. Foram incluídos estudos primários observacionais e experimentais, com adultos (18 a 65 anos) que recebessem apoio social de instituição religiosa e que apresentassem ao menos um dos domínios da atividade física. Os estudos foram localizados independentemente do ano de publicação e nos idiomas inglês, português e espanhol. Dos 195 estudos identificados nas bases de dados, 35 atendiam os critérios de inclusão e sete apresentavam algum resultado sobre a relação entre a religiosidade, o apoio social e a atividade física. Os artigos foram publicados entre 2004 e 2013 e todos foram realizados nos Estados Unidos. Quanto ao desenho de estudo, cinco estudos eram transversais e dois controlados não randomizados. Em conclusão, pessoas mais assíduas às igrejas/instituições religiosas estão mais propensas a iniciarem e se engajarem em atividades físicas. $\mathrm{O}$ apoio social obtido através das instituições religiosas parece não ser suficiente para promover a atividade física em níveis recomendados.

\section{Palavras-chave}

Religião; Apoio social; Atividade física; Revisão.

\begin{abstract}
The purpose was to map the literature on the relationship of social support arising from religious institutions and physical activity. The electronic searches were conducted in August 2014 in the electronic databases MEDLINE via PubMed, EMBASE, CENTRAL (Cochrane Central Register of Controlled Trials), LILACS and PsychINFO. Observational and experimental primary studies were included, with adults (18-65 years) who received social support from religious institution and provided at least one of the domains of physical activity. Studies were located independent of publication year and in English, Portuguese and Spanish. Of 195 studies identified in databases, 35 met the inclusion criteria and seven had result on the relationship between religiosity, social support and physical activity. The articles were published between 2004 and 2013 and all were performed in the United States. Regarding the study design, five studies were cross-sectional and two nonrandomized controlled. In conclusion People more assiduous to the churches / religious institutions are more likely to initiate and engage in physical activities. Social support obtained through religious institutions seems to be insufficient to promote physical activity at recommended levels.

Keywords

Religion; Social Support; Motor Activity; Review.
\end{abstract}

\section{Introdução}

Estima-se que a cada três adultos, um é fisicamente inativo e isso globalmente ${ }^{1}$. A inatividade física causa de 6 a 10\% das principais doenças não transmissíveis

\footnotetext{
1 Universidade Federal do Paraná, Programa de Pós-graduaçăo em Educação Física, Curitiba/PR - Brasil.

2 Grupo de Pesquisa em Atividade Física e Qualidade de Vida - GPAQ - Curitiba/ PR - Brasil

3 Universidade Federal de São Paulo - UNIFESP, Programa de Pós-Graduação em Saúde Baseada em Evidência, São Paulo/SP - Brasil.

4 Pontifícia Universidade Católica do Paraná - Programa de Pós-graduação em Planejamento Urbano, PUCPR, Curitiba/PR - Brasi

5 Centro Cochrane do Brasil, São Paulo/SP - Brasil.
}

(doenças coronarianas, diabetes tipo 2 e câncer de cólon e mama) e 9\% das mortes prematuras. Em 2008 foi responsável por mais de 5,3 das 57 milhões de mortes $^{2}$. Esta combinação torna a inatividade física um dos mais importantes problemas de saúde pública no mundo ${ }^{3}$.

Entretanto, conceber a atividade física somente em termos de prevenção de doenças é um equívoco. Os benefícios que a atividade física pode proporcionar a saúde estão bem evidenciados na literatura científica ${ }^{4,5}$ e abrangem outros aspectos além da saúde fí- 
sica: bem estar mental, senso de propósito, melhor qualidade de vida, melhora do sono, redução do estresse, bem como, relações sociais mais fortes e conectadas ${ }^{2}$.

$\mathrm{O}$ apoio social, entendido como a ajuda ou assistência recebida através das relações sociais e interpessoais ${ }^{6}$ é evidenciado como um importante fator de influência para a promoção da atividade física ${ }^{7,8}$ independente da intensidade e da modalidade $e^{9}$ entre idosos, adultos e adolescentes ${ }^{8}$. Relacionamentos próximos, incluindo membros da família, amigos e colegas de trabalho, auxiliam as pessoas a engajarem-se em atividades físicas de lazer ${ }^{10}$.

O interesse sobre a relação entre o apoio social e religiosidade cresceu nos últimos anos. Pessoas envolvidas com ambiente religioso (ex: frequentam cultos, missas e grupos religiosos), possuem maior rede de apoio, mais contatos e mais satisfação com o apoio recebido ${ }^{11}$. Neste sentido, a atividade religiosa se configura como uma importante fonte de apoio social ${ }^{11}$, dado que aumenta a qualidade e quantidade das redes sociais.

Apesar deste potencial, as evidências sobre a relação entre apoio social advindo das instituições religiosas apresenta associação consistente com maiores níveis de atividade física ${ }^{12}$, ainda que tais instituições tenham efetivo papel no apoio social geral ${ }^{13}$. Portanto, o presente trabalho pretende sintetizar a literatura existente sobre apoio social, religiosidade e atividade física, e analisar em que medida o apoio social advindo de instituições religiosas influencia a prática de atividade física. O entendimento desta relação pode ser útil na criação e desenho de intervenções mais eficazes para promover atividade física, tendo instituições religiosas como possíveis canais de intervenção, e emprego de grupos religiosos como eventual ferramenta para a promoção de atividade física ${ }^{3}$.

\section{Métodos}

O mapeamento da literatura foi realizado seguindo os seguintes critérios de inclusão: estudos com participantes adultos (entre 18 e 65 anos); que reportassem apoio social de uma instituição religiosa; e que incluísse ao menos um dos domínios da atividade física (lazer, transporte, atividades domésticas e ocupacionais). Foram incluídos apenas estudos primários observacionais e experimentais.

A busca foi realizada em Agosto de 2014, nas bases de dados eletrônicas Medline via Pubmed, EMBASE, CENTRAL (Cochrane Central Register of Controlled Trials), LILACS e PsychINFO. Uma estratégia de busca matriz para a base de dados Medline foi realizada e posteriormente adaptada para as outras bases. Os descritores-chave da estratégia de busca foram: "religião", "apoio social" e "atividade física”; os descritores similares e os derivados foram também adicionados para aumentar a sensibilidade da busca. Os revisores também realizaram buscas manuais das listas de referências dos estudos incluídos e de outras revisões localizadas que eram relacionadas ao tema. Os estudos foram localizados independentemente do ano de publicação e idioma.

Os estudos identificados foram avaliados quanto à elegibilidade por meio da leitura dos títulos e resumos. Os estudos elegíveis foram avaliados em texto completo sendo incluídos aqueles que atenderam aos critérios de inclusão preestabelecidos. O processo de avaliação da elegibilidade e inclusão dos estudos foi realizado por dois avaliadores independentes e as discordâncias foram solucionadas em reunião de consenso. Os mesmos avaliadores realizaram a extração dos dados utilizando um formulário padronizado com informações sobre: identificação do estudo, objetivo, tipo de estudo, tamanho e características da amostra, medidas de religiosidade, apoio social e atividade física e principais resultados. Os resulta- 
dos foram apresentados de maneira descritiva através de uma tabela dos estudos incluídos (tabela 1).

\section{Resultados}

No fluxograma (figura 1) estão apresentados os dados de seleção de artigos para cada uma das fases da revisão. Dos 203 estudos identificados 8 estavam duplicados entre as bases. Após leitura dos títulos e resumos 35 estudos foram considerados elegíveis para a revisão (concordância entre avaliadores $=87,18 \%$ ). Após leitura dos artigos na integra sete cumpriam os critérios de inclusão estabelecidos (concordância entre avaliadores $=80 \%$ ).
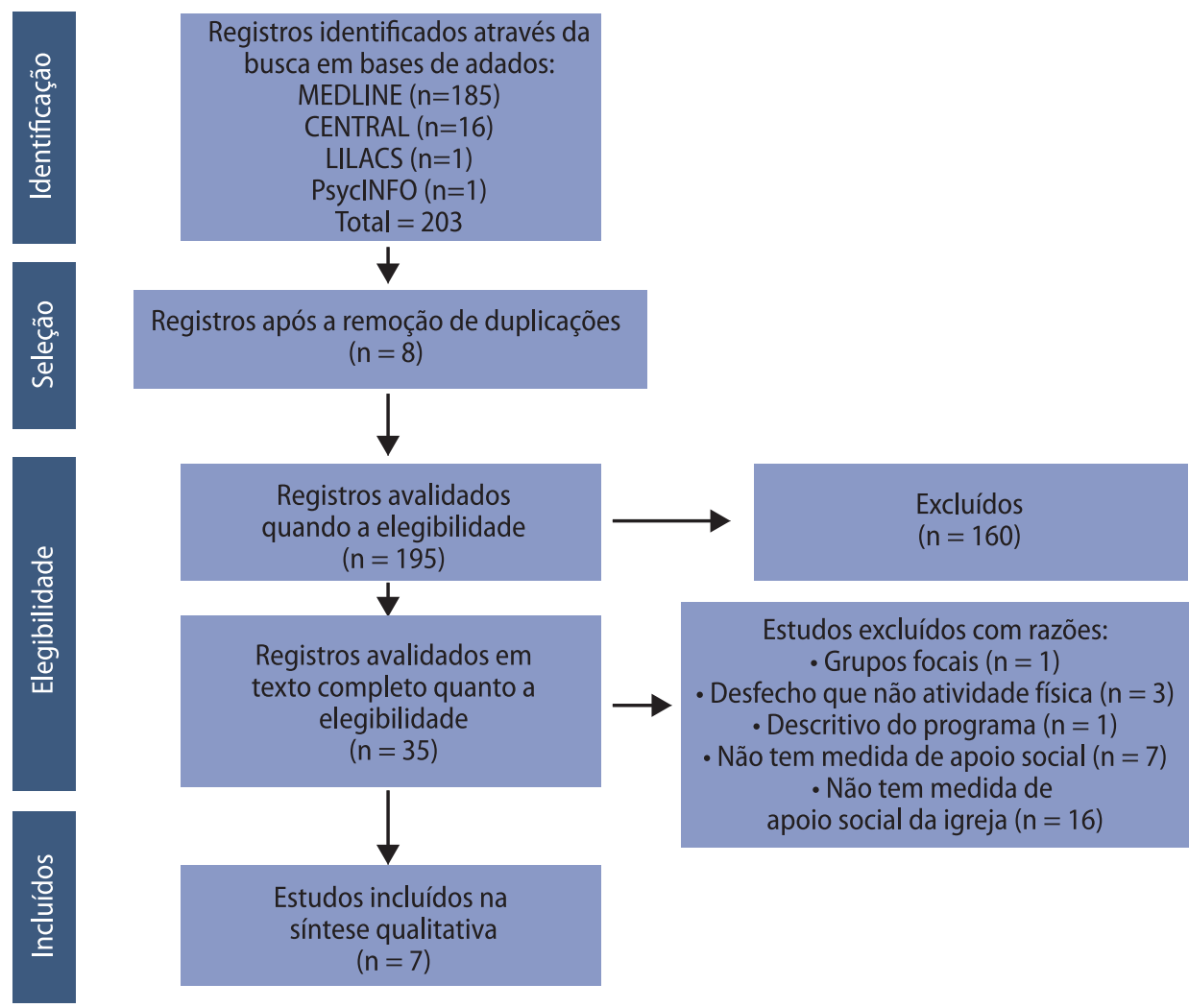

FIGURA 1 - Fluxograma do processo de identificação, seleção e inclusão dos estudos.

Os estudos foram publicados entre 2004 e 2013. Todos estudos foram realizados nos Estados Unidos. Quanto à população incluída, cinco estudos foram realizados com afro americanos, um com comunidade rural e um estudo com a população em geral. Em todos eles, o sexo feminino teve maior representação entre os participantes (61 a 81\%). Quanto ao desenho metodológico, cinco estudos foram transversais e dois controlados não randomizados. Em relação a medida de atividade física, 5 estudos utilizaram medida autorrelatada, três utilizou medida objetiva (acelerômetro) e três utilizou medidas combinadas (autorrelatada e pedômetro).

Em relação as medidas do apoio social advindo da igreja (quadro 1), três estudos reportaram apoio instrumental (igreja patrocina programas de atividade físi$\mathrm{ca}^{12}$; igreja oferece programas ${ }^{14,15}$ ), quatro estudos apoio informativo (líder da igreja aconselha a começar ou continuar caminhada ou exercícios/igreja inclui mensagens sobre saúde e atividade física nos boletins semanais ${ }^{12}$; diretor de saúde ou outros membros falaram sobre a participação em um programa de atividade física ${ }^{16}$; membros da igreja te orientam ser mais ativo ${ }^{14}$; pastor ou lideranças da igreja falam 
sobre atividade física na igreja/mensagens nos boletins informativos, folhetos e quadro de avisos15), quatro estudos apoio emocional (proximidade da relação com Deus ${ }^{17}$; recebeu encorajamento de alguém da igreja para participar em um programa de atividades físicas/exercícios físicos ${ }^{14,16}$; pessoas da igreja falam sobre a importância da atividade física ${ }^{14}$; pessoas da igreja te escutam falar sobre seus problemas e preocupações/você faz as pessoas da sua igreja se sentirem amadas e cuidadas/ pessoas da sua igreja estão dispostas a te ajudar quando esta doente $\left.{ }^{18}\right)$. Em dois estudos não foi possível identificar a classificação (membros da igreja se envolviam em atos favoráveis a atividade física ${ }^{19}$; apoio positivo e negativo da igreja ${ }^{18}$ ).

QUADRO 1 - Classificação do apoio social advindo da igreja de acordo com classificação do apoio social relacionado a saúde

\begin{tabular}{|c|c|c|c|c|c|c|c|}
\hline & $\mathrm{Kim}^{17}$ & $K^{K a n u^{12}}$ & Bopp $^{19}$ & Bopp $^{16}$ & Baruth $^{14}$ & Debnam $^{18}$ & Baruth $^{15}$ \\
\hline Informativo & & $x$ & & $x$ & $x$ & & $x$ \\
\hline \multicolumn{8}{|l|}{ Avaliativo } \\
\hline Instrumental & & $x$ & & & $x$ & & $x$ \\
\hline Emocional & $x$ & & & $x$ & $x$ & $x$ & \\
\hline Não identificado* & & & $x$ & & & $x$ & \\
\hline
\end{tabular}

*As informações disponíveis no artigo eram insuficientes para identificar a classe do apoio social avaliado.

TABELA 2 - Descrição dos estudos incluídos.

\begin{tabular}{|c|c|c|c|c|c|c|c|c|}
\hline ID & Amostra & Idade & País & Objetivo & $\begin{array}{l}\text { Desenho } \\
\text { Metodológico }\end{array}$ & $\begin{array}{l}\text { Medida de apoio social } \\
\text { da igreja }\end{array}$ & $\begin{array}{l}\text { Medida de } \\
\text { Atividade física }\end{array}$ & Principais resultados \\
\hline $\operatorname{Kim}^{17}$ & $\begin{array}{l}546 \\
(353 \\
\text { mulheres, } \\
193 \\
\text { homens) }\end{array}$ & $\begin{array}{l}\text { Mulheres } \\
(44 \pm 20,5) \\
\text { Homens } \\
(42 \pm 20,8)\end{array}$ & EUA & $\begin{array}{l}\text { Examinar } \\
\text { associação entre } \\
\text { religião, ingestão } \\
\text { de gordura e AF } \\
\text { tendo o apoio } \\
\text { social como } \\
\text { mediador }\end{array}$ & Transversal & $\begin{array}{l}\text { Apoio social: divino } \\
\text { (quão próxima é sua } \\
\text { relação com Deus), } \\
\text { e da congregação } \\
\text { (apoio social positivo e } \\
\text { negativo) }\end{array}$ & $\begin{array}{l}\text { Autorrelatado } \\
\text {-itens sobre } \\
\text { AF moderada e } \\
\text { vigorosa }\end{array}$ & $\begin{array}{l}\text { Entre os homens o } \\
\text { apoio social divino (mais } \\
\text { oração) foi associado com } \\
\text { maior AF moderada }\end{array}$ \\
\hline Kanu $^{12}$ & $\begin{array}{l}1625(81,7 \% \\
\text { mulheres, } \\
18,3 \% \\
\text { homens) }\end{array}$ & $\begin{array}{l}18-29 \\
(16,4 \%) ; \\
30-44 \\
(20,4 \%) ; \\
45-64 \\
(36,1 \%) ; \\
65+(27,1 \%)\end{array}$ & EUA & $\begin{array}{l}\text { Testar a associação } \\
\text { entre o apoio } \\
\text { instrumental e } \\
\text { informacional } \\
\text { advindos da } \\
\text { igreja e a AF em } \\
\text { residentes de } \\
\text { comunidades } \\
\text { rurais }\end{array}$ & Transversal & $\begin{array}{l}\text { Apoio social } \\
\text { informacional: } \\
\text { conselhos, sugestões } \\
\text { e informações, Ex: líder } \\
\text { da igreja aconselhou a } \\
\text { começar ou continuar } \\
\text { caminhada ou } \\
\text { exercícios; Igreja inclui } \\
\text { mensagens sobre } \\
\text { saúde e AF nos boletins } \\
\text { semanais; } \\
\text { Apoio social } \\
\text { instrumental: programas } \\
\text { de AF que a igreja } \\
\text { patrocina }\end{array}$ & $\begin{array}{l}\text { Autorrelatado - } \\
\text { cumpre ou não as } \\
\text { recomendações } \\
\text { de AF (30min de } \\
\text { AF moderada } 5 \\
\text { dias na semana) }\end{array}$ & $\begin{array}{l}\text { Associação entre apoio } \\
\text { social instrumental e } \\
\text { realização de alguma AF } \\
\text { mas não entre as duas } \\
\text { formas de apoio social e } \\
\text { cumprir a recomendação } \\
\text { de AF: grupo de } \\
\text { caminhada patrocinado } \\
\text { pela igreja (OR = 2,19; } \\
\text { 95\% Cl, 1,13-4,25), grupos } \\
\text { de exercício (OR = 1,95; } \\
\text { 95\% Cl, 1,08-3,52), outros } \\
\text { programas de exercício } \\
\text { (OR = 2,76; } 95 \% \mathrm{Cl}, 1,17- \\
6,53)\end{array}$ \\
\hline Bopp ${ }^{19}$ & $\begin{array}{l}146 \\
(117 \\
\text { mulheres, } 29 \\
\text { homens) }\end{array}$ & $\begin{array}{l}\text { Intervenção } \\
(52,5 \pm 13,8) \\
\text { Comparação } \\
(52 \pm 12,7)\end{array}$ & EUA & $\begin{array}{l}\text { Avaliar a } \\
\text { efetividade de } \\
8 \text { semanas de } \\
\text { uma intervenção } \\
\text { comportamental } \\
\text { na AF }\end{array}$ & $\begin{array}{l}\text { Controlado } \\
\text { não } \\
\text { randomizado }\end{array}$ & $\begin{array}{l}\text { Escala de Sallis et } \\
\text { al.: os participantes } \\
\text { classificaram a } \\
\text { frequência do } \\
\text { envolvimento da família, } \\
\text { amigos e membros } \\
\text { da igreja em atitudes } \\
\text { favoráveis a AF nos } \\
\text { últimos } 3 \text { meses }\end{array}$ & $\begin{array}{l}\text { AF autorrelatado. } \\
\text { (CHAMPs } \\
\text { modificado; } \\
\text { recordatório por } \\
\text { uma semana); } \\
\text { Gasto energético } \\
\text { (calculado a partir } \\
\text { do CHAMPs); } \\
\text { Mensuração } \\
\text { objetiva: } \\
\text { pedômetro }\end{array}$ & $\begin{array}{l}\text { Efeito significativo para } \\
\text { a interação tempo x } \\
\text { grupo para o apoio social } \\
\text { advindo dos membros } \\
\text { da igreja }(P=0,03), \text { com } \\
\text { maior aumento no apoio } \\
\text { social para o grupo de } \\
\text { intervenção; } \\
\text { Após } 3 \text { meses, grande } \\
\text { tamanho de efeito }(0,92) \\
\text { e após } 6 \text { meses médio } \\
\text { tamanho de efeito }(0,59) \\
\text { foram observados para o } \\
\text { apoio social dos membros } \\
\text { da igreja na direção da } \\
\text { hipótese }\end{array}$ \\
\hline
\end{tabular}




\begin{tabular}{|c|c|c|c|c|c|c|c|c|}
\hline ID & Amostra & Idade & País & Objetivo & $\begin{array}{l}\text { Desenho } \\
\text { Metodológico }\end{array}$ & $\begin{array}{l}\text { Medida de apoio social } \\
\text { da igreja }\end{array}$ & $\begin{array}{l}\text { Medida de } \\
\text { Atividade física }\end{array}$ & Principais resultados \\
\hline Bopp ${ }^{16}$ & $\begin{array}{l}571(71 \% \\
\text { mulheres, } \\
29 \% \\
\text { homens) }\end{array}$ & $\begin{array}{l}18-34 \\
(10,5 \%) \\
35-49 \\
(33,3 \%) ; \\
50-64 \\
(31,6 \%) ; \\
65-74 \\
(13,8 \%) ; \\
75+(0,8 \%)\end{array}$ & EUA & $\begin{array}{l}\text { Determinar o grau } \\
\text { de envolvimento } \\
\text { da igreja na } \\
\text { promoção da AF } \\
\text { de seus membros }\end{array}$ & Transversal & $\begin{array}{l}\text { Participantes foram } \\
\text { questionados sobre: se } \\
\text { já conversaram com o } \\
\text { Diretor de Saúde da sua } \\
\text { Igreja ou com outros } \\
\text { membros da lgreja } \\
\text { sobre a participação } \\
\text { em um programa de } \\
\text { AF, e se já receberam } \\
\text { encorajamento de } \\
\text { alguém da Igreja para } \\
\text { participar em um } \\
\text { programa de AF. }\end{array}$ & $\begin{array}{l}\text { Autorrelatado: } \\
\text { BRFSS CDC } \\
\text { 2001: frequência } \\
\text { e duração de } \\
\text { AF moderada, } \\
\text { vigorosa, } \\
\text { caminhada. } \\
\text { Classificação: } \\
\text { cumpre a } \\
\text { recomendação, } \\
\text { insuficientemente } \\
\text { ativo e inativo }\end{array}$ & $\begin{array}{l}\text { Os que relataram existir } \\
\text { programas de AF na } \\
\text { igreja (42\%) estavam } \\
\text { mais propensos a cumprir } \\
\text { a recomendação de AF. } \\
\text { Entre os que relataram } \\
\text { participar não foi } \\
\text { observada associação } \\
\text { com AF. Não houve } \\
\text { relação significativa } \\
\text { entre o diretor de saúde } \\
\text { falar sobre AF e cumprir } \\
\text { as recomendações. O } \\
\text { apoio social interpessoal } \\
\text { foi significativamente } \\
\text { associado com o } \\
\text { cumprimento das } \\
\text { recomendações de AF. } \\
\text { Os participantes com } \\
\text { menor pontuação de } \\
\text { apoio interpessoal foram } \\
\text { os menos propensos a } \\
\text { atender a recomendação } \\
\text { (24,3\%). Os que relataram } \\
\text { ao menos um tipo de } \\
\text { apoio ou ambos os } \\
\text { tipos estavam mais } \\
\text { propensos a cumprir } \\
\text { recomendações de } \\
\text { AF (35,8\% e } 36,4 \% \text {, } \\
\text { respectivamente). }\end{array}$ \\
\hline Baruth $^{14}$ & $\begin{array}{l}418(71 \% \\
\text { mulheres, } \\
29 \% \\
\text { homens) }\end{array}$ & $54 \pm 17$ & EUA & $\begin{array}{l}\text { Examinar os } \\
\text { mediadores } \\
\text { psicossociais } \\
\text { (autoeficácia, } \\
\text { gosto para } \\
\text { exercício e apoio } \\
\text { da igreja) de uma } \\
\text { intervenção com } \\
\text { base religiosa } \\
\text { de AF }\end{array}$ & $\begin{array}{l}\text { Controlado } \\
\text { não } \\
\text { randomizado }\end{array}$ & $\begin{array}{l}\text { Cinco itens designados } \\
\text { a capturar aspectos } \\
\text { do apoio da igreja } \\
\text { (encorajamento e } \\
\text { apoio dos membros } \\
\text { para ser fisicamente } \\
\text { ativo e aumentar } \\
\text { oportunidades para ser } \\
\text { fisicamente ativo) }\end{array}$ & $\begin{array}{l}\text { Autorrelatado } \\
\text { via telefone: AF } \\
\text { ocupacional, } \\
\text { caminhada e } \\
\text { AF moderada e } \\
\text { vigorosa (modulo } \\
\text { do sistema de } \\
\text { vigilância para } \\
\text { fatores de risco } \\
\text { comportamentais } \\
\text { - BRFSS) }\end{array}$ & $\begin{array}{l}\text { Todas as medidas de } \\
\text { apoio social da igreja } \\
\text { foram significativamente } \\
\text { maiores no início do } \\
\text { estudo em comparação } \\
\text { com o grupo que } \\
\text { recebeu a intervenção } \\
\text { posteriormente. A } \\
\text { intervenção teve um } \\
\text { efeito marginalmente } \\
\text { significativo no aumento } \\
\text { do apoio instrumental } \\
\text { recebido da igreja } \\
p=0,08 \text {. }\end{array}$ \\
\hline Debnam ${ }^{18}$ & $\begin{array}{l}2370 \text { ( } 61,8 \% \\
\text { mulheres, } \\
38,2 \% \\
\text { homens) }\end{array}$ & $53,6 \pm 14,8$ & EUA & $\begin{array}{l}\text { Examinar a } \\
\text { contribuição } \\
\text { relativa do apoio } \\
\text { social para uma } \\
\text { variedade de } \\
\text { comportamentos } \\
\text { saudáveis em } \\
\text { uma amostra } \\
\text { probabilística } \\
\text { nacional de } \\
\text { homens e } \\
\text { mulheres }\end{array}$ & Transversal & $\begin{array}{l}\text { Instrumento: medida } \\
\text { multidimensional } \\
\text { da religiosidade/ } \\
\text { espiritualidade para } \\
\text { pesquisa em saúde. }\end{array}$ & $\begin{array}{l}\text { Autorrelatado: } \\
\text { IPAQ abreviado e } \\
\text { recordatório de } \\
7 \text { dias }\end{array}$ & $\begin{array}{l}\text { Somente a AF moderada } \\
\text { foi significativamente } \\
\text { associada com o apoio } \\
\text { social e com apoio social } \\
\text { antecipado ( } r=0,07, p \\
<0,05 \text { ). } \\
\text { Para a AF moderada o } \\
\text { envolvimento religioso } \\
\text { demonstrou uma } \\
\text { significante contribuição } \\
\left.\text { adicional ( } \triangle R^{2} P<0,05\right) \text {, } \\
\text { com o apoio antecipado } \\
\text { sendo positivamente } \\
\text { associado (P }<0,05) \text {. O } \\
\text { nível de apoio social } \\
\text { não foi associado com a } \\
\text { AF, entretanto aqueles } \\
\text { com apoio religioso } \\
\text { antecipado engajam-se } \\
\text { mais em AF moderada } \\
\text { que os recebem em } \\
\text { menor quantidade esse } \\
\text { tipo de apoio }\end{array}$ \\
\hline
\end{tabular}




\begin{tabular}{|c|c|c|c|c|c|c|c|c|}
\hline ID & Amostra & Idade & País & Objetivo & $\begin{array}{l}\text { Desenho } \\
\text { Metodológico }\end{array}$ & $\begin{array}{l}\text { Medida de apoio social } \\
\text { da igreja }\end{array}$ & $\begin{array}{l}\text { Medida de } \\
\text { Atividade física }\end{array}$ & Principais resultados \\
\hline Baruth ${ }^{15}$ & $\begin{array}{l}309 \text { (78\% } \\
\text { mulheres } \\
\text { e } 22 \% \\
\text { homens) }\end{array}$ & $\begin{array}{l}54 \pm 12,3 \\
\text { anos }\end{array}$ & EUA & $\begin{array}{l}\text { Examinar a relação } \\
\text { entre ambiente } \\
\text { percebido, apoio } \\
\text { social recebido da } \\
\text { igreja para AF e } \\
\text { comportamentos } \\
\text { de atividade física }\end{array}$ & Transversal & $\begin{array}{l}\text { Sete itens com uma } \\
\text { escala de resposta } \\
\text { de quatro pontos } \\
\text { (raramente a todo } \\
\text { tempo) para avaliar o } \\
\text { apoio para AF durante } \\
12 \text { meses. A escala foi } \\
\text { nomeada: instrumental, } \\
\text { informacional verbal, } \\
\text { informacional escrito e } \\
\text { apoio total da igreja }\end{array}$ & $\begin{array}{l}\text { Mensuração } \\
\text { objetiva: } \\
\text { Acelerômetro } \\
\text { Actigraph (GT1M) }\end{array}$ & $\begin{array}{l}\text { O apoio total, } \\
\text { informacional verbal e } \\
\text { escrito recebido da igreja } \\
\text { foi significativamente } \\
\text { associado com maiores } \\
\text { níveis de AF leve e } \\
\text { menores níveis de } \\
\text { comportamento } \\
\text { sedentário entre os } \\
\text { homens. A relação entre } \\
\text { apoio informacional } \\
\text { verbal e escrito e AF } \\
\text { moderada e vigorosa } \\
\text { aproximou-se da } \\
\text { significância para os } \\
\text { homens, mas não para } \\
\text { mulheres. O apoio } \\
\text { social instrumental } \\
\text { não foi associado com } \\
\text { o comportamento } \\
\text { fisicamente ativo }\end{array}$ \\
\hline
\end{tabular}

AF: atividade física.

\section{Discussão}

Os estudos na área de religiosidade e saúde constituem um linha de pesquisa em constante crescimento e consolidação ${ }^{11,21}$. Apesar de os estudos sobre a influência do apoio social para a atividade física datarem da década de 1980, é recente o interesse sobre a influência do apoio social advindo de instituições religiosas para a atividade física, o que também pode ser observado nas datas dos artigos aqui apresentados (2004 a 2013).

Os resultados mostram que seis dos sete manuscritos identificados foram realizados com populações específicas (cinco com afro-americanos e um com comunidades rurais) e todos nos Estados Unidos. Uma possível explicação é que a incidência e risco de mortalidade por doenças do coração, câncer e diabetes é desproporcionalmente maior entre os afro-americanos, quando comparados aos americanos europeus ${ }^{22}$. Outro aspecto diz respeito a missão das igrejas que historicamente tem sido consideradas "a pedra angular" entre os afro-americanos, as quais estenderam sua missão para questões sociais, econômicas e políticas que têm afetado essas comunidades ${ }^{23}$. As instituições religiosas parecem ser importantes parceiras para a redução das disparidades relacionadas a saúde, em especial para populações consideradas difíceis de alcançar. Podem implementar programas e atividades que são culturalmente relevantes, adaptadas espiritualmente e adequarse de acordo com os desejos e necessidades de sua congregação particular ${ }^{23}$.

A baixa representação de homens nas amostras dos estudos revisados (variando de 18 a 38\% das amostras descritas) é comum em intervenções e pode ser particularmente comum em estudos com base religiosa/espiritual, pois as mulheres estão mais propensas que homens a frequentarem igreja ${ }^{15,24}$. Entretanto, é importante considerar que a constatação de que mulheres são "mais religiosas" que homens tem como base pesquisas com amostras Cristãs. Quando observado outros grupos, como Hindus e muçulmanos, a relação é inversa o que reflete as normas culturais em questão ${ }^{25}$.

Em relação ao apoio social, alguns autores consideram quatro dimensões relacionadas a saúde: instrumental (apoio tangível incluindo produtos e serviços), informativo (aconselhamentos, sugestões e informações), avaliativo (informações 
úteis para autoavaliação) e emocional (sentimento de confiança, carinho, amor e empatia $)^{12,20}$. As medidas de apoio social advindos da igreja e relatadas nos manuscritos não se distanciaram das dimensões de apoio social relatados na literatura como relacionados a saúde. $\mathrm{O}$ apoio social interpessoal, mais especificamente, conversar com outras pessoas da igreja sobre atividade física foi associado com o cumprimento da recomendação de atividades físicas ${ }^{16}$. É comum que as pessoas que participam de instituições religiosas compartilhem as mesmas crenças e valores. Discutir temas de saúde, como atividade física com membros da igreja oferece uma oportunidade para o apoio social informacional e possivelmente emocional ${ }^{26}$.

Em quatro dos sete estudos, o apoio social advindo de instituições religiosas foi relacionado com realizar algum tipo de atividade física leve e/ou atividades físicas de intensidade moderada ${ }^{12,15,17,18}$. Sabe-se que a promoção da atividade física, mesmo que de leve intensidade, é benéfica para a saúde ${ }^{27-29}$, além de ser um ponto de partida realista para uma população pouco ativa fisicamente.

O apoio social informativo oferecido pela igreja (disponibilização de informações no quadro de avisos, boletins, apostilas e etc., sermão/pregação sobre a importância da atividade física) tem potencial para alcançar um grande número de pessoas, mesmo aquelas com menor predisposição a participar dos programas ${ }^{15}$. Uma estratégia relatada por Campbell e colaboradores, em estudo de revisão, aponta que pessoas podem ser mais receptivas para mensagens com referenciais espirituais e bíblicas, do que para referenciais técnicos ${ }^{23}$.

O presente estudo, de caráter descritivo, não avaliou a qualidade metodológica dos estudos selecionados. Os pesquisadores não tiveram acesso a outras bases eletrônicas, como por exemplo o EMBASE, onde a literatura europeia é amplamente disseminada. Assim sendo, os resultados apresentados não podem ser generalizados globalmente. A maioria dos estudos revisados eram de delineamento transversal e mesmo sendo capazes de evidenciar associações entre variáveis, a relação de causalidade entre o apoio social advindo da igreja e o aumento dos níveis de atividade física não pode ser assumida. Dentre os estudos experimentais, estes eram controlados, porém, não randomizados. Os resultados encontrados nestes ensaios vão ao encontro com a hipótese inicial, porém, estudos com maior rigor metodológico são necessários.

As investigações mostram relações positivas entre a religiosidade e o uso de drogas licitas e ilícitas ${ }^{30,31}$, saúde mental nas áreas de depressão, abuso de drogas e suicídio $^{32}$, satisfação com a vida ${ }^{11,33}$ e felicidade ${ }^{11}$. Não obstante, o elevado percentual de pessoas com afiliação religiosa é um indicativo da importância da religiosidade na vida das pessoas ${ }^{34,35}$.

Apesar das limitações e dificuldades de comparação entre os estudos, é possível considerar que o apoio social para atividade física advindo de instituições religiosas parece ser importante para o início e/ou engajamento atividades físicas, mas não o suficiente para promover a atividade física em níveis recomendados.

\section{Referências}

1. Hallal PC, Andersen LB, Bull FC, Guthold R, Haskell W, Ekelund U, et al. Global physical activity levels: surveillance progress, pitfalls, and prospects. Lancet. 2012;380(9838):247-57.

2. Lee IM, Shiroma EJ, Lobelo F, Puska P, Blair SN, Katzmarzyk PT, et al. Effect of physical inactivity on major non-communicable diseases worldwide: an analysis of burden of disease and life expectancy. Lancet. 2012;380(9838):219-29.

3. Sallis JF. Physical Activity \& Behavioral Medicine. Behavioral Medicine \& Health Psycology 3. London:SAGE; 1999. 210 p. 
4. Warburton DE, Nicol CW, Bredin SS. Health benefits of physical activity: the evidence. CMAJ. 2006;174(6):801-9.

5. Committee PAGA. Physical Activity Guidelines Advisory Committee Report 2008. Washington: United States: Department of Health and Human Services; 2008.

6. Glanz K, Rimer BK, Viswanath K. Health behavior and health education: theory, research, and practice. 4th, editor. San Francisco: Jossey-Bass; 2008.

7. Peterson JA, Yates BC, Atwood JR, Hertzog M. Effects of a Physical Activity Intervention for Women. West J Nurs Res. 2005;27(1):93-110.

8. Bauman AE, Reis RS, Sallis JF, Wells JC, Loos RJF, Martin BW. Correlates of physical activity: Why are some people physically active and others not? Lancet. 2012;380(9838):258-71.

9. Wendel-Vos W, Droomers M, Kremers S, Brug J, van Lenthe F. Potential environmental determinants of physical activity in adults: a systematic review. Obes Rev. 2007;8(5):425-40.

10. Carron A V, Hausenblas HA, Mack DE. Social influence and exercise: a meta-analysis. J Sport Exerc Psychol. 1996;18(1):1-16.

11. Koenig HG, McCullough ME. Handbook of religion and health. New York: Oxford university press; 2001.

12. Kanu M, Baker E, Brownson RC. Exploring associations between church-based social support and physical activity. J Phys Act Heal. 2008;5(4):504-15.

13. Peterson J, Atwood JR, Yates B. Key elements for church-based health promotion programs: outcome-based literature review. Public Heal Nurs. 2002;19(6):401-11.

14. Baruth M, Wilcox S, Blair S, Hooker S, Hussey J, Saunders R. Psychosocial mediators of a faith-based physical activity intervention: implications and lessons learned from null findings. Heal Educ Res. 2010;25(4):645-55.

15. Baruth M, Wilcox S, Saunders RP, Hooker SP, HusseyJR, Blair SN. Perceived environmental church support and physical activity among Black church members. Health Educ Behav. 2013;40(6):712-20.

16. Bopp M, Wilcox S, Laken M, Mcclorin L. Physical activity participation in African American churches. J Cult Divers. 2009;16(1).

17. Kim KH, Sobal J. Religion, social support, fat intake and physical activity. Public Heal Nutr. 2004;7(6):773-81.

18. Debnam K, Holt CL, Clark EM, Roth DL, Southward P. Relationship between religious social support and general social support with health behaviors in a national sample of African Americans. J Behav Med. 2012;35(2):179-89.

19. Bopp M, Wilcox S, Laken M, Hooker SP, Parra-Medina D, Saunders R, et al. 8 Steps to Fitness: a faith-based, behavior change physical activity intervention for African Americans. J Phys Act Heal. 2009;6(5):568-77.

20. Glanz K, Lewis FM, Rimer BK. Health behavior and health education: theory, research, and practice. 2nd, editor. San Francisco: Jossey-Bass; 1996. 487 p.

21. Lucchetti G, Lucchetti ALG. Spirituality, Religion, and Health: Over the Last 15 Years of Field Research (1999-2013). Int J Psychiatry Med. 2014;48(3):199-215.

22. Services UD of H and H, Cdc. Health, United States, 2008, special feature on health of young adults. Natl Cent Heal Stat. 2009; Available from: www.cdc.gov/nchs/data/hus/

23. Campbell MK, Hudson MA, Resnicow K, Blakeney N, Paxton A, Baskin M. Church-based health promotion interventions: evidence and lessons learned. Annu Rev Public Health. United States; 2007;28:213-34.

24. Neri MC, Melo LCC de. Novo Mapa das Religiões (New Map of Religions) - DOI: 10.5752/P.2175-5841.2011v9n23p637. HORIZONTE. 2011.

25. Loewenthal KM, MacLeod AK, Cinnirella M. Are women more religious than men? Gender differences in religious activity among different religious groups in the UK. Pers Individ Dif. 2002;32(1):133-9.

26. Eng E, Hatch J, Callan A. Institutionalizing social support through the church and into the community. Health Educ Q. 1985;12(1):81-92.

27. Demakakos P, Hamer M, Stamatakis E, Steptoe A. Low-intensity physical activity is associated with reduced risk of incident type 2 diabetes in older adults: Evidence from the English Longitudinal Study of Ageing. Diabetologia. 2010;53(9):1877-85.

28. Herzig K-H, Ahola R, Leppäluoto J, Jokelainen J, Jämsä T, Keinänen-Kiukaanniemi S. Light physical activity determined by a motion sensor decreases insulin resistance, improves lipid homeostasis and reduces visceral fat in high-risk subjects: PreDiabEx study RCT. Int J Obes (Lond). 2013;(November 2013):1-8. 
29. Gando Y, Yamamoto K, Murakami H, Ohmori Y, Kawakami R, Sanada K, et al. Longer time spent in light physical activity is associated with reduced arterial stiffness in older adults. Hypertension. 2010;56(3):540-6.

30. Gomes FC, de Andrade AG, Izbicki R, Almeida AM, de Oliveira LG. Religion as a protective factor against drug use among Brazilian university students: A national survey. Rev Bras Psiquiatr. 2013;35(1):29-37.

31. Kub J, Solari-Twadell PA. Religiosity/spirituality and substance use in adolescence as related to positive development: a literature review. J Addict Nurs. 2013;24(4):247-62.

32. Bonelli RM, Koenig HG. Mental Disorders, Religion and Spirituality 1990 to 2010: A Systematic Evidence-Based Review. J Relig Health. 2013;52(2):657-73.

33. Berthold A, Ruch W. Satisfaction with life and character strengths of non-religious and religious people: it's practicing one's religion that makes the difference. Front Psychol. 2014;5(August):1-9.

34. IBGE IBDGEE-. Dados completos sobre a Religião no Brasil - IBGE - Censo 2010 [Internet]. Censo Demográfico 2010. 2010. Available from: ftp:/ftp.ibge.gov.br/ Censos/Censo_Demografico_2010/Caracteristicas_Gerais_Religiao_Deficiencia/ caracteristicas_religiao_deficiencia.pdf

35. Affiliation R, February D. U . S . Religious Landscape Survey About the Pew Forum on Religion \& Public Life. Landscape. 2008;(February):1-207.

ENDEREÇO PARA

CORRESPONDÊNCIA

MARIANA S. REIS

silva.mariana@pucpr.br
Rua Imaculada Conceição, 1155 ,

CEP 80215-901, Prado Velho,

Curitiba - PR, BR.

Tel: 3271-2503.
RECEBIDO

REVISADO

$01 / 11 / 2015$

APROVADO $18 / 12 / 2015$ 